\title{
Rep:grid Software Supported Visualization of a Corporate Culture
}

\author{
Henning Bundtzen, Gerriet Hinrichs \\ Szent István University, Kaposvár, Hungary
}

\begin{abstract}
This paper shows arrestingly how a corporate culture can be analyzed and visualised in a three-dimensional sphere using a repertory grid-based software. A theory that builds personal assessment criteria of individuals is leveraged through IT application to consolidate results of numerous employees and leaders. The software in this case study processed 21.114 unique graded construct-elementratings creating a comprehensive data basis which allows the visualization of an entity's corporate culture.
\end{abstract}

Keywords - repertory grid software, corporate culture, personal construct theory, generalized Procrustes analysis

\section{Introduction}

Corporate culture (CC) is a complex system of numerous norms, values and perceptions formed through events. Common approaches to analyze CC include qualitative research methods consisting of how and why questions without producing quantitative results [1]. The creation of an interpretable visualization of $\mathrm{CC}$ though requires a combination of qualitative descriptions with quantitative data that contextualizes the descriptive perceptions.

Thus, a triangulation approach to $\mathrm{CC}$ is required to gather qualitative and quantitative data simultaneously.

DOI: $10.18421 /$ TEM103-12

https://doi.org/10.18421/TEM103-12

Corresponding author: Henning Bundtzen, Szent István University, Kaposvár, Hungary. Email: henning.bundtzen@outlook.de

Received: 26 March 2021.

Revised: 05 July 2021.

Accepted: 10 July 2021.

Published: 27 August 2021.

(c) BY-NC-ND(C) 2021 Henning Bundtzen \& Gerriet Hinrichs; published by UIKTEN. This work is licensed under the Creative Commons Attribution-NonCommercial-NoDerivs 4.0 License.

The article is published with Open Access at www.temjournal.com
One possibility to access the culture of an organization can be personal construct psychology (PCP) formulated by George A. Kelly who stated that "A person's processes are psychologically channelized by the ways in which he anticipates events" [2]. With the above statement George A. Kelly initiated his theory of PCP. The theory describes a psychological approach to the subjective view of how people make sense of the world around them [3].

If Kelly is correct this theory is capable of supplying a completely new approach to investigate organizational behavior, specifically corporate culture (CC), as the culture of an organization reflects a group of people forming their subjective views by engaging the same organizational experiences [4],[5]. When Kelly formulated his theory though computer technology it was not at a stage to offset grid data from numerous participants to create a unified grid from several people of one organization. Manually this process can neither be undertaken.

To describe a new approach for analyzing $\mathrm{CC}$ the underlying study deploys repertory grid structured interviews to derive corporate culture related constructs from employees and leaders. The results are bipolar constructs on whose basis all elements that represent the organization are rated.

Statistical methodology generalized Procrustes analysis (GPA) is applied, which enables a threedimensional visualization of the interview results [6], [7]. In addition to the graphical visualizations statistical results using element to construct cluster relations are produced. Making an entire corporate culture visible three-dimensionally, interpretable, and mathematically analyzable would hold a significant contribution of this research study with regards to science and practice in itself. The results though are unknown as the qualitative assessment criteria are not set by the researcher, but they are rather defined in the process of the interview by the participant [8]. This bears the risk of eliciting inexplicable group results if no distinct cultural pattern is created by the generated data. On the other side, this uncertainty induces the potential of creating new corporate cultural perspectives that have not been researched [4]. 


\section{Methodology}

Collecting data within the framework of personal construct psychology offers several possibilities. For this study software that supported repertory grid interviews (RGI) were chosen. Originating from a clinical context in psychology RGIs have developed into an application technique in various research areas, including organizational behavior and corporate culture [9]. Distinct to seldomly quantitative questionnaires or qualitative interviews RGIs produce simultaneously qualitative statements which are quantitatively evaluated by the interviewee. That way a certain feature of an employee world is translated into a cognitive map through a grid [7]. As a precondition, a list of objects (called elements) is determined based on the domain of interest. These elements are presented to the interviewee to carry out an elicitation process. The RGI technique records the bipolar constructs in a data matrix which allows carrying out statistical and mathematical calculations on the peculiarity of values and norms [9].

In general, two types of repertory grid can be distinguished. Firstly, a common grid in which not only the elements but also the constructs are predefined [10]. As a result, this defaults the qualitative aspect of personal construct psychology, possibly to constrain the research scope. Common grid distances between objects and attributes can be defined in a statistical procedure by cluster analysis [10]. In a unique grid the opposite approach is chosen, neither elements nor constructs are predefined or kept constant throughout the interview process. While in common grids the interviewer determines strongly the context and qualitative aspects to be rated, in a unique grid the subject and qualitative attributes are determined by the interviewee [11]. For this research study a mixture between common and unique grid is chosen:

1. Elements are predefined, to ensure that attributes in association with corporate culture are derived.

2. Constructs are created and rated by the proband to prevent limitation or exertion of influence by the researcher.

This approach was selected as the study tries to stand in the shoes of an employee of the organization and excerpting their thoughts and images of the world surrounding them [11]. But to limit this imagination to the organizational context, excluding attributes about the private situation, the research elements need to be predefined. Still, as the elements are rated on the basis of the constructs the mathematical relationship between the interviewees attributes form the cultural picture of an organization. This way RGI based on personal construct psychology are a powerful way to generate qualitative data of an employee's attitudes, emotions and experiences of the organization they work for [7]. As well as PCP, the repertory grid technique has been applied to numerous business context including work surroundings, employment and learning assessments which highlights the techniques flexibility [12]. In order to classify important issues from the final list of elicited constructs, a widely used analytical approach is content analysis by creating clusters [13]. Content analysis attempts to group features into the same category with a common meaning. The groups themselves can be predefined (which was not the case in this repertory grid study) or from the list of elicited construct obtained inductively [9].

\subsection{Description of the Case Study}

As a basis for this research 61 repertory grid structured interviews were carried out within an organization. The research object is a wholesale company in the consumer industry with approximately 500 employees from which 21 staff members are in leadership roles. Table 1 indicates the structure of the participants who carried out the repertory grid structured interviews for this case study:

\section{Table 1. Participant structure}

\begin{tabular}{|c|c|c|c|}
\hline Gender & \multicolumn{3}{|c|}{ No. of employers } \\
\hline male & 37 & 0 - 1 employer & 17 \\
\hline female & 24 & $2+$ employer & 44 \\
\hline Age & \multicolumn{3}{|c|}{ Education } \\
\hline 21-30 years & 10 & no training & 0 \\
\hline $31-40$ years & 20 & vocational & 37 \\
\hline $41-50$ years & 24 & Bachelor & 9 \\
\hline $51-60$ years & 6 & Master & 16 \\
\hline$>60$ years & 1 & $\mathrm{PhD} /$ doctor & 0 \\
\hline Job tenure & \multicolumn{3}{|c|}{ Department } \\
\hline less 1 year & 8 & Transport logistics & 3 \\
\hline$>1-5$ years & 10 & Internal services & 3 \\
\hline$>5-10$ years & 11 & Warehouse & 15 \\
\hline$>10-20$ years & 22 & Marketing & 3 \\
\hline$>20$ years & 10 & Internal logistics & 2 \\
\hline \multicolumn{4}{|c|}{ Department (continued) } \\
\hline$\overline{\mathrm{CEO}}$ & 2 & Procurement & 4 \\
\hline Sales national & 9 & IT & 3 \\
\hline Export sales & 8 & Accounts & 1 \\
\hline Product mgmt. & 6 & Personnel & 2 \\
\hline
\end{tabular}

The company is led by two general managers who besides the founder are also shareholders of the organization. The company has experienced 45 years of growth and accomplished 200 Mio. $€$ turnover in

2018. The forecast and current business development is positive and a turnover increase is forecasted for 2019 and 2020. The pandemic crisis in 
2020 has affected the organization considerably, but not negatively, rather leading to an unexpected grow of sales, and difficulties to obtain sufficient goods from oversees and fast changing customer requirements were triggered by different lockdown scenarios in various countries. Overall, the organization is financially healthy, paying above standard pay scale. Employees have the possibility to invest into a shareholding company so that they directly participate from the success of the corporation. Fluctuation among employees is at a comparably low level. Recruitment of additional staff is still unproblematic and applicants state that they have been told about the great working atmosphere. Hence a high employer attractiveness is presumed.

\subsection{Determination of the Sample Size}

The determination of the samples size for repertory grid studies can be an issue because of the triangulation research approach including qualitative and quantitative elements. Consequently, the two main aims of the interviews and content analysis have to be considered. First, the researcher wants to cover the majority of the research domains inherent topics [9]. These are the clusters that are created by the combination of mathematical and content analysis. Second, a minimum number of constructs is contained in each cluster to enable statistical analysis of the repertory grid data set [9]. One possible option is to conduct additional interviews until a saturation of subcategories of organizational culture is reached. In other words, until no further clusters are created by the additional construct elicited through the added interview [14]. This requires that between each interview the cluster analysis is conducted. Other studies are based on a rule of thumb which proposes 15 to 25 repertory grid interviews to cover any domain of interest [15]. These assumptions are made because the quantity of elicited constructs per subtopic, the probability and even the overall amount of clusters are undetermined before conduction of the interviews. Nevertheless, a possible approach can be making expectations about the required number of categories based on a literature review [9].

An approach for an ex-ante determination of the required sample size suggested by Heckmann \& Burk [9] is based on the probability assumption of receiving at least a set percent of the total subject of which each contains at least a minimum number of constructs. This approach combines the two main research aims of this study and facilitates a predefined sample size. To create a holistic picture of the corporate culture of the research object shown in Table 2 lists the input criteria that have been determined for this investigation:
Table 2. Input settings for determination of the sample size

\begin{tabular}{lc}
\hline \multicolumn{1}{c}{ Type of input setting } & Projection \\
\hline Expected constructs per interview: & $8-16$ \\
Type of probability (figure 3): & Normal \\
Expected mean: & distribution \\
Standard deviation: & 12 \\
Expected average of constructs: & 1 \\
Type of probability (figure 1) & 12 \\
Targeted probability: & exponential \\
Clusters / categories: & $95 \%$ \\
Min. number of constructs per cat. & 10 \\
\hline
\end{tabular}

The number of expected constructs per interview is based on the targeted interview length of approx. 120 minutes. All interviews were scheduled for this time frame and rarely any is shorter than the scheduled two hours. Based on the above setting Figures 1 and 2 show which probabilities were calculated as preconditions for the calculation of the final sample size.

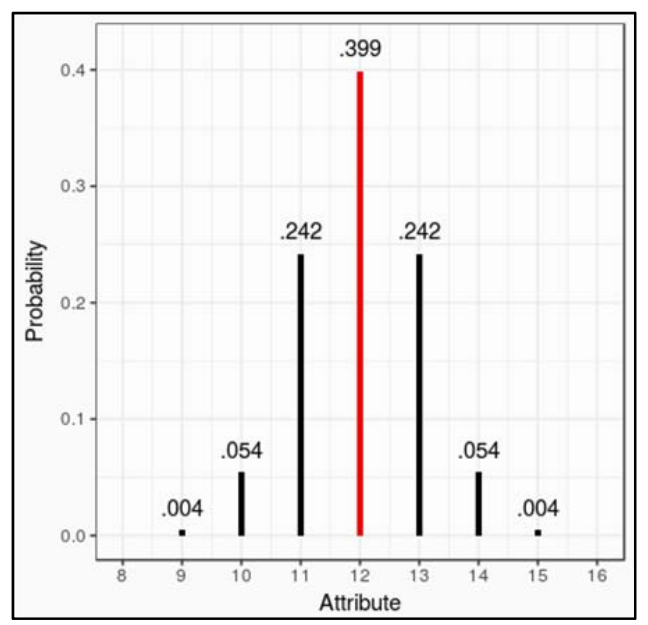

Figure 1. Probability for number of attributes per interview

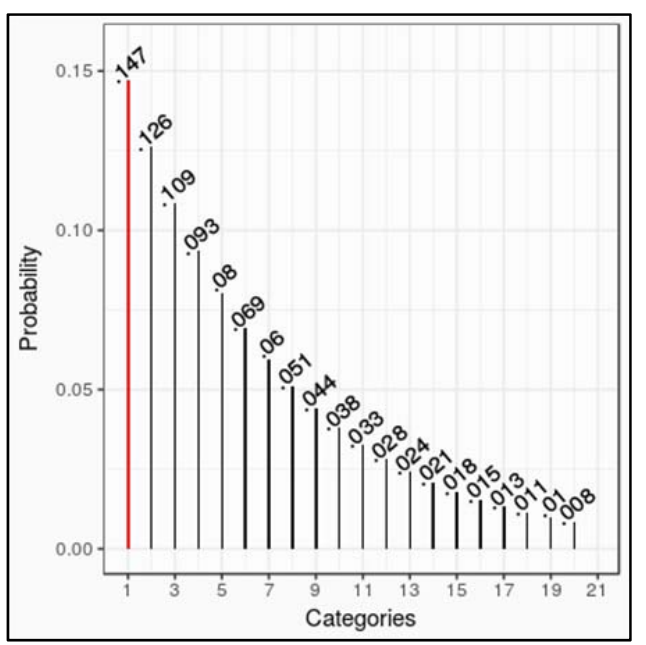

Figure 2. Probability for number of categories per interview 
For the determination of the sample size a probability of $95 \%$ of covering all inherent topics was targeted. A test was run for probabilities of $90 /$ 95 and $99 \%$ in combination with a minimum number of 8,9 or 10 attributes or constructs per category. The following graph shows that on basis of these criteria a minimum sample size of $\mathrm{N}=60$ is required to achieve at least 20 clusters with a minimum count of 10 constructs per cluster to be evaluated as selfcontained topic within the corporate culture context of the investigate object. As 21 people were in leadership positions the final sample size of $\mathrm{N}=61$ was determined with included 21 managers plus 40 employees across all departments of the organization. That way data with direct context to a leadermember-exchange was generated.

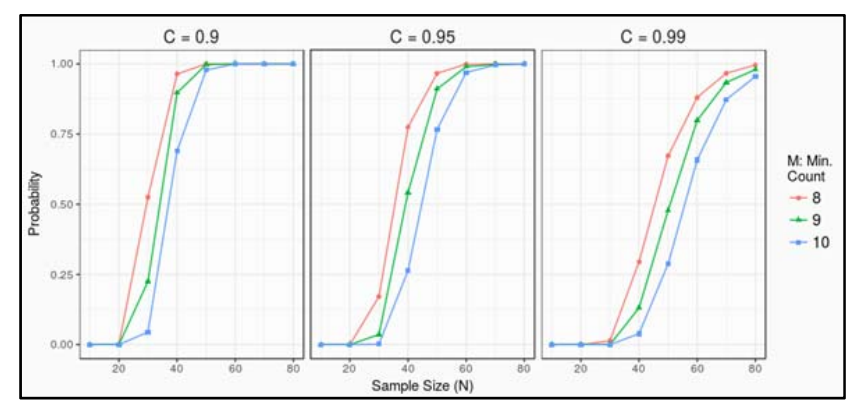

Figure 3. Determination of the sample size

In ex-post consideration this approach was quite conservative as the final number of clusters derived was 36 of which each holds at least 13 individual assessment criteria.

\subsection{Conduction of the Interviews}

The following describes the four different reiterating phases of the interviews. In the first phase from the set of 27 elements a triad of three elements are presented to the interviewee (Figure 4), asking to select which two elements are similar but different from the third one:

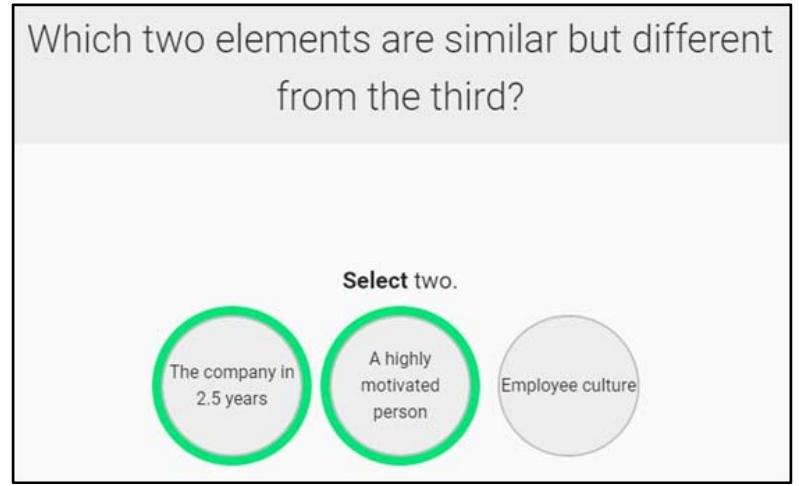

Figure 4. Phase 1 of the interview - Triad comparison
Once the proband selected two intuitively phase, two is designated to elicit the first personal construct related to the selected two elements which reflect the organizational culture of the entity under investigation. The interviewee is asked what makes the two elements similar and at the same time different from the third one as indicated in Figure 5.

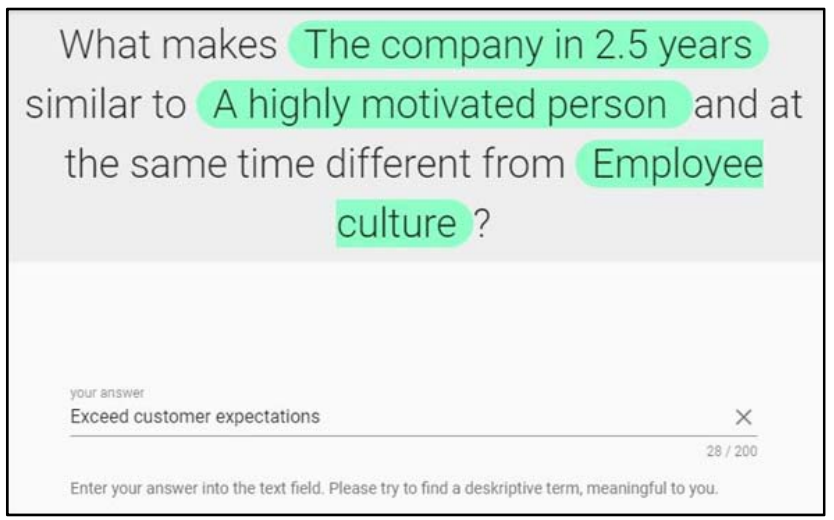

Figure 5. Phase 2 of the interview - Elicitation of the first construct

After elicitation of the first, qualitative construct formulated by the interviewee in which system asks for the opposite assessment criteria of this construct (Figure 6). This bipolarity of constructs enables in a later step the visualization of quantitative results in repertory grids.

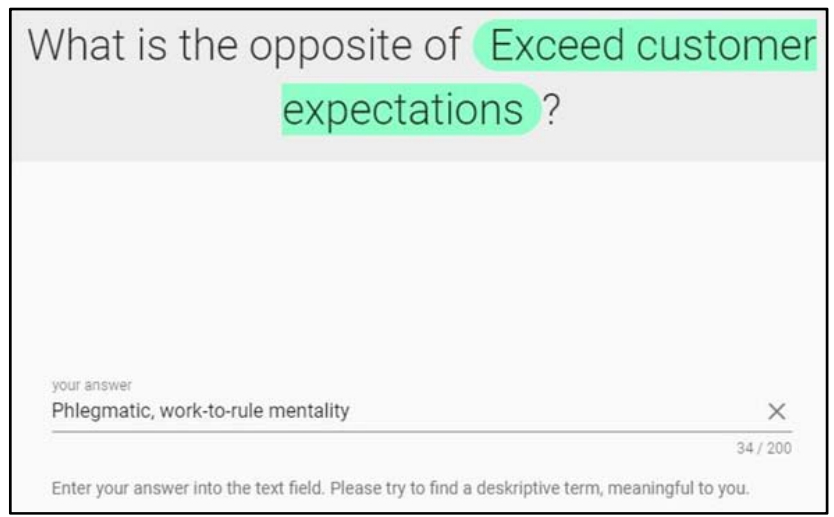

Figure 6. Phase 3 of the interview - Opposite construct creation

In the final step the proband is presented a tetrapolar field in which all 27 elements have to be rated, there is a possibility to leave elements unrated in case of no intuitive evaluation possibility. That way not only the qualitative assessment criteria in form of personal constructs are elicited to develop a holistic picture of the underlying corporate culture, but quantitative data in relation to the personal assessment criteria is produced on a scale from 0 to 100. The interviewee has the possibility to assign only one characteristic by choosing the specification "exceed customer needs $=0$ " and "phlegmatic, workto-rule mentality $=100$ " for the elements. In addition, any combination with the other two extremes 
"neither" or "both" can be selected for each element. The following picture shows exemplified software's view of the interviewee with rating examples of the utilized corporate elements. These components are put by drag and drop into the tetra-polar field, highlighting the exact rating scale at the edges of the rating frame. During the interview the rated elements were hidden, to ensure an intuitive rating without rethinking the evaluation after placing additional elements.

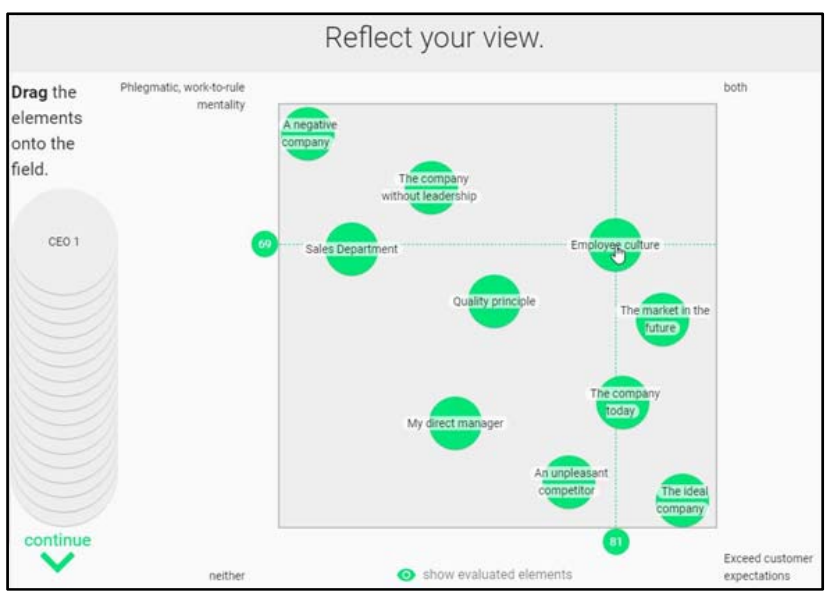

Figure 7. Phase 4 of the interview - Rating of the elements

Once all elements were rated the proband returned to phase 1 with a different triad of elements. That way a new set of bipolar constructs is elicited. The role of the researcher is to support the interviewees creative thinking in case the person has difficulties with a certain triad or finding the opposite to derived construct which can occur due to the difficulty evoked by triadic comparisons [7]. The researcher should not influence the constructs through any questions aimed at provoking a certain construct, but rather by supporting the inductive or deductive thinking with leading interviewee to specific events or common concepts with laddering questions like "What do you mean by ..." [7]. This depends on the probands general approach to make sense (inductively or deductively). In sum the iterations throughout 61 qualitative interviews produced 782 personal constructs. These multiplied by 27 element ratings result in 21.114 unique graded constructelement-ratings building, which is a comprehensive data basis to analyze and visualized the entity's corporate culture.

\subsection{Generalized Procrustes Analysis (GPA) for 3D visualization}

Inherently repertory grid structured interviews are a qualitative research methodology as probands enter its own wordings based on their interpretation of the organization. The utilized computer software creates, in the reflection phase, in-depth data based on the entries of the participant [6]. Statistically this data set can be transformed using Generalized Procrustes Analysis (GPA) to create from the sum of individual grids an aggregated grid which allows to visualize the corporate culture. In the first step the interviewees' different usage of scales are adjusted to a collective central mapping sheet [16], and this process is identified as translation. Afterwards, variations grounded in the distinct utilization of attributes by the interviewees are eradicated by reflection and rotation until a maximum agreement is achieved by applying Procrustes rotation technique [16], [17]. This process is followed by scaling to unit variance through shrinking and stretching the configuration size aiming at an equalization of different ranges of the scale displayed in Figure 7. This is done without altering the relative distances between the elements [18]. Provided that the mean, rotation, and scale reflect individual variations of subordinate significance for the explanation of element discrepancies, generalized Procrustes analysis seems an appropriate statistical tool for investigating repertory grid data. Relative distances between elements are kept which is essential to the visualization and statistical based interpretation of research results in this study [18]. After application of GPA each element and construct have a unique set of coordinates, thus enabling the researcher to create clusters and interpret the relative distances of element and construct clusters. The spatial distances reflect the groups cultural associations within the organization. The following Figure 8 shows the entire data set including the most relevant elements, all clusters and construct locations indicated as dots.

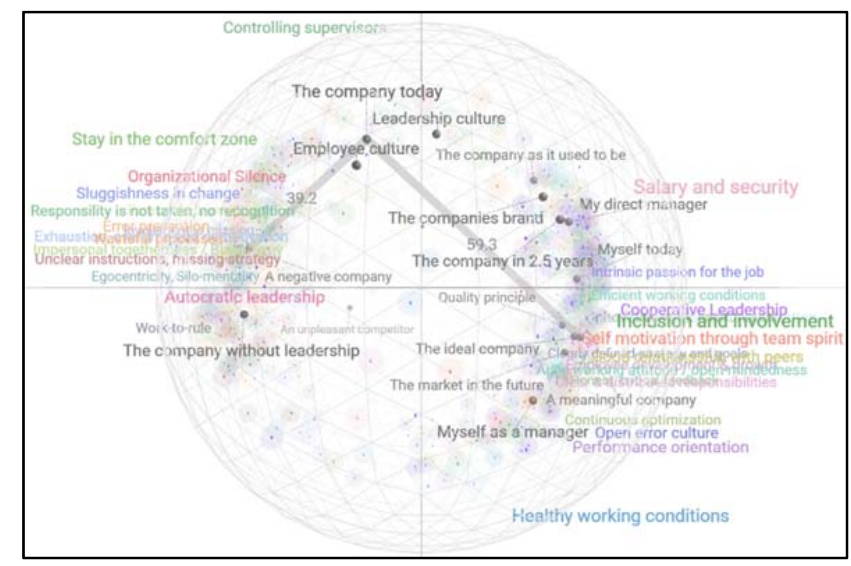

Figure 8. Visualized data set after GPA application

Visualizing and analyzing 782 constructs in one graph or even statistically would result in unclear outcome. As several attributes has similar or identical meaning, an aggregation into clusters with its own heading seems an appropriate approach to explore this data set on corporate culture. In the first step the system created an initial set of clusters based on the spatial location of all constructs. This set was 
reviewed and extended by the researcher semantically to ensure that related constructs are combined in one cluster. Feixas et al. (2002) explored in their study the degree on consensual accordance between researcher in case an identical data set of attributes and elements was qualitatively dissected independently from each other. The agreement resulted in $87.3 \%$ of consensus [5]. Consequently, the conduction of content analysis aiming at the creation of clusters was added to the methodological approach of this study.

The spatial distances (from a maximum of 100) between the clusters and elements representing the organization allow an analysis of the corporate culture. Minor distance in this setup represents a high degree of association of an element with a cluster. The next chapter was built on these circumstances in order to explore the data set under different focuses with regards to the content.

\section{Discussion}

$\mathrm{CC}$ is a complex framework of a diverse number of people's assumptions and interpretations. Making these visible via an unbiased methodology enables the discovery of new paths in research of organization behavior. The utilized software, rep:grid, has proven to be a strong support in this investigation, both in the data generation and analysis. The dataset can be extracted including all 782 constructs and their unique grid coordinates. In this manner the data can be analyzed later with additional tools such as R.

The explorative approach to analyze organizational culture, combined with reviews of contemporary literature, revealed to be a research approach worth following. In particular this holds true for triangulation methods that generate quantitative and qualitative data simultaneously. In this case the limitation of one investigated organization had the opposite advantage that explicitly Leader-member exchange-related data was created and analyzed. Here the intuitive and unbiased approach of PCP proved advantageous as it obviates socially desirable results by leaders and followers.

The most relevant dichotomy of this study refers to the dataset being limited to one organization. On the one hand, two factors favor this limitation of scope: direct LMX data, which enabled new implications, such as the results with regard to self-perception in leadership, and the possibility of creating a holistic $\mathrm{CC}$ view of an organization, since a conflation of repertory grid data from several organizations would not have generated expedient results. On the other hand, making enquiries with interviewees of several companies would have made comparable results, which would lower the representativeness of the organization. In addition, the depths and number of clusters or topics would decrease with a reduction of interviews per organization, and still the results would not be generalizable in a quantitative manner. As a consequence an in-depth qualitative approach was chosen with a limitation to one organization. In retrospect this has proven to be an effectual method as new paths in organizational research were identified. To make the qualitative results of this research which combined empirical and qualitive elements generally applicable, quantitative enquiries would need to be performed. In this manner the exploratory approach developed several research paths.

In particular, implications result from the findings in the context of error management and selfperception in leadership. The quantitative and qualitative data created empirically in this study guided the literature review, which made evident that further research is required in this field due to its importance in the framework of CC.

\section{Conclusion}

Repertory grid structure interviews supported by a GPA based software can be a strong tool to investigate and visualize corporate culture. From the created data set several conclusions and further research implications were withdrawn. The software used in this case study (rep:grid) is able to produce instant 3D graphs directly after the interview. That way a mutual consensus with the interviewee can be obtained.

The research approach indicates that through exploratory methods, new insights in organizational research can be created. Of course, these have to be verified and substantiated in a quantitative matter, but the data generated through RGIs offers the first basis to motivate and further research in this direction is worthwhile. The triangulation research model of this study, which included generating quantitative and qualitative results simultaneously with a subsequent analysis from a different perspective, has proven to be a powerful tool in organizational research.

In summary PCP combined with RGIs is identified as a suitable tool and method to analyze the CC. In practical usage in particular, it has a prime advantage over questionnaire-driven investigations. No stakeholder can lead the investigation in a certain direction by in- or excluding specific cultural elements. As Kelly highlighted, people construe their own perceptions of the world or organization on how they anticipate events. This reflects a filter when conducting a questionnaire or interview, which is eliminated by the unassured and unprejudiced approach of PCP. In practical terms this may 
however reflect an obstacle as leaders and stakeholders have to commit to an investigation of their $\mathrm{CC}$ without knowing in advance the assessments, or even the assessment criteria. As a consequence, a commitment for this approach and the uncertain results is required, just in the same way as it was required in the initiation of this research. In retrospect, however, we can confirm that it has been worth all the doubts and efforts.

\section{References}

[1]. Gray, B., Stensaker, I. G., \& Jansen, K. J. (2012). Qualitative challenges for complexifying organizational change research: Context, voice, and time. The Journal of applied behavioral science, 48(2), 121-134.

[2]. Kelly, G. A. (1955). The psychology of personal constructs. Volume 1: A theory of personality. WW Norton and Company.

[3]. Senior, B., \& Swailes, S. (2004). The dimensions of management team performance: a repertory grid study. International Journal of Productivity and Performance Management, 53(4), 317-333.

[4]. Brophy, S. (2007). PCP in business: A spectrum of experience. Personal Construct Theory \& Practice, 4, 57-62.

[5]. Feixas, G., Geldschläger, H., \& Neimeyer, R. A. (2002). Content analysis of personal constructs. Journal of constructivist psychology, 15(1), 1-19.

[6]. Mak, A. H., Lumbers, M., Eves, A., \& Chang, R. C. (2013). An application of the repertory grid method and generalised Procrustes analysis to investigate the motivational factors of tourist food consumption. International Journal of Hospitality Management, 35, 327-338.

[7]. Mark, E. S., Richard, T., \& David, H. (1996). Using repertory grids in management. Journal of European Industrial Training, 20(3), 3-30.
[8]. Fransella, F., Bell, R., \& Bannister, D. (2004). A manual for repertory grid technique. John Wiley \& Sons.

[9]. Heckmann, M., \& Burk, L. (2017). Gridsampler-A simulation tool to determine the required sample size for repertory grid studies. Journal of Open Research Software, 5(1).

[10]. Fransella, F. E. (2005). The essential practitioner's handbook of personal construct psychology. John Wiley \& Sons Ltd.

[11]. Hill, A. D., White, M. A., \& Wallace, J. C. (2014). Unobtrusive measurement of psychological constructs in organizational research. Organizational psychology review, 4(2), 148-174.

[12]. Bourne, D., \& Jankowicz, D. A. (2018). The repertory grid technique. In Qualitative methodologies in organization studies (pp. 127-149). Palgrave Macmillan, Cham.

[13]. Hauser, M., Jonas, K., \& Riemann, R. (2011). Measuring salient food attitudes and food-related values. An elaborated, conflicting and interdependent system. Appetite, 57(2), 329-338.

[14]. Napier, N. P., Keil, M., \& Tan, F. B. (2009). IT project managers' construction of successful project management practice: a repertory grid investigation. Information Systems Journal, 19(3), 255-282.

[15]. Tan, F. B., \& Hunter, M. G. (2002). The repertory grid technique: a method for the study of cognition in information systems. MIS Quarterly, 26(1), 39-57.

[16]. Grice, J. W., \& Assad, K. K. (2009). Generalized procrustes analysis: a tool for exploring aggregates and persons. Applied Multivariate Research, 13(1), 93-112.

[17]. Gower, J. C. (1975). Generalized procrustes analysis. Psychometrika, 40(1), 33-51.

[18]. Tomic, O., Berget, I., \& Næs, T. (2015). A comparison of generalised procrustes analysis and multiple factor analysis for projective mapping data. Food quality and preference, 43, 34-46. 